\section{Support of New Triage Protocol among Acute Stroke Care Providers}

\author{
Haitham M. Hussein ${ }^{a}$ David C. Anderson ${ }^{b}$ \\ a Regions Hospital Comprehensive Stroke Center, Saint Paul, MN, USA; \\ ${ }^{b}$ Department of Neurology, University of Minnesota, Minneapolis, MN, USA
}

\section{Keywords}

Acute stroke · Prehospital care · Emergency medical service - Large vessel occlusion .

Mechanical thrombectomy $\cdot$ Intravenous thrombolysis

\begin{abstract}
Objective: We conducted an online survey to gauge the acceptance of sending acute stroke patients with suspected large vessel occlusion (LVO) directly to an endovascular-capable hospital $(\mathrm{ECH})$ even if that means bypassing a closer alteplase-capable hospital (ACH) without endovascular capability. Methods: The survey was composed of two cases of acute stroke, one with cortical symptoms suggestive of LVO and the other without. In each case, responders were asked to choose between triaging to a closer $\mathrm{ACH}$ or an $\mathrm{ECH}$ that is further away and to provide an opinion regarding the maximum extra travel time they would tolerate if they chose the $\mathrm{ECH}$. The survey was sent electronically to national groups of neurologists, emergency department (ED) physicians, emergency medical service (EMS) directors, and stroke coordinators. Results: There were 320 responders from 44 states, most of them with 10 years or more of experience. Most of the responders, $72.5 \%$, chose ECH for the LVO case, while $56 \%$ chose ACH for the non-LVO case. There were marked differences in responses by specialty: neurology strongly supported ECH for LVO and strongly supported ACH for non-LVO, most ED and EMS chose ECH for both cases, and stroke coordinators were the least supportive of bypassing $\mathrm{ACH}$. Almost all groups agreed on $30 \mathrm{~min}$ as the acceptable extra transfer time to $\mathrm{ECH}$. Conclusion: Among the survey responders, there is a broad acceptance of the idea of bypassing ACH and going straight to ECH when LVO is suspected; however, there is less agreement on triaging patients with non-LVO stroke.




\section{Background}

Sixteen years is how long it took after the first proof-of-concept randomized clinical trial for endovascular treatment of stroke due to large vessel occlusion (LVO) to appear in the national guidelines [1,2]. A meta-analysis of recent endovascular trials showed strong benefit overall among eligible subjects and in subgroups traditionally thought not to benefit or be at high risk of hemorrhagic complications, such as patients aged 70 years or older and patients with severe stroke (NIHSS $>20$ ) [3]. Looking at them separately [4-8] or after pooling individual patient data [9], the endovascular trials show that the shorter the time to treatment, the better the outcome. In fact, the most expeditious trial, ESCAPE [6], was the only one to show mortality benefit despite being prematurely terminated before reaching its target sample size.

The current model of triaging patients with suspected stroke to the nearest IV alteplasecapable hospital $(\mathrm{ACH})$ that does not have endovascular capability leads to inevitable delay for LVO patients as subsequent transport to an endovascular-capable hospital (ECH) is necessary. Delay may decrease the benefit of the procedure or preclude performing it if the patient arrives at the ECH after the treatment window has closed. One proposed model of triaging LVO patients to the nearest ECH even if it means bypassing a closer ACH, has been extensively debated for the last 2 years [10]. We conducted an online survey to gauge attitudes towards this proposed model among medical specialties involved in acute stroke management.

\section{Method}

The survey featured two cases of acute stroke with witnessed onset and paramedics arriving at the scene within the first hour from symptom onset. One patient had cortical signs (aphasia and gaze deviation) suggestive of LVO and the other hemiparesis and hemihypesthesia without cortical signs. For each, the responders were asked to choose whether to send the patient to the nearest ACH or the nearest ECH, if the latter is further, and to provide the maximum acceptable extra transport time to get to the ECH. Responders were instructed to select the option they think is in the best interest of the patient, regardless of their current practice.

The survey was created on the SurveyMonkey website (www.surveymonkey.com) and was electronically sent to: (a) the American Academy of Neurology (AAN) stroke/vascular neurology section online community, (b) the AAN endovascular/interventional neurology section online community, (c) listserve of emergency department (ED) physicians practicing in 3 hospitals affiliated with our academic institution (2 of them are level I trauma centers), (d) listserve of the American College of Emergency Physicians - Emergency Medical Service (EMS) physicians group, (e) the stroke centers coordinators in the state of Minnesota, and the coordinators of comprehensive stroke centers nationwide. The survey allowed the responders to specify other specialties or if still in training. Responses were collected for 3 weeks. Participants were not asked for and did not provide any personal identifying data. Participants were informed that the data would be presented publicly in an aggregated fashion. Participation was voluntary and consent was implied.

\section{Results}

There were 320 responses: 90 neurologists, 86 ED physicians, 61 EMS physicians, 35 stroke centers coordinators, 16 interventional neuroradiologists, 9 trainees (of any of the above-mentioned specialties), and 3 intensivists. Most responses were from the United States spanning 44 states. Half the sample practiced in an academic setting, half in a private setting, and $58 \%$ had more than 10 years of experience. 


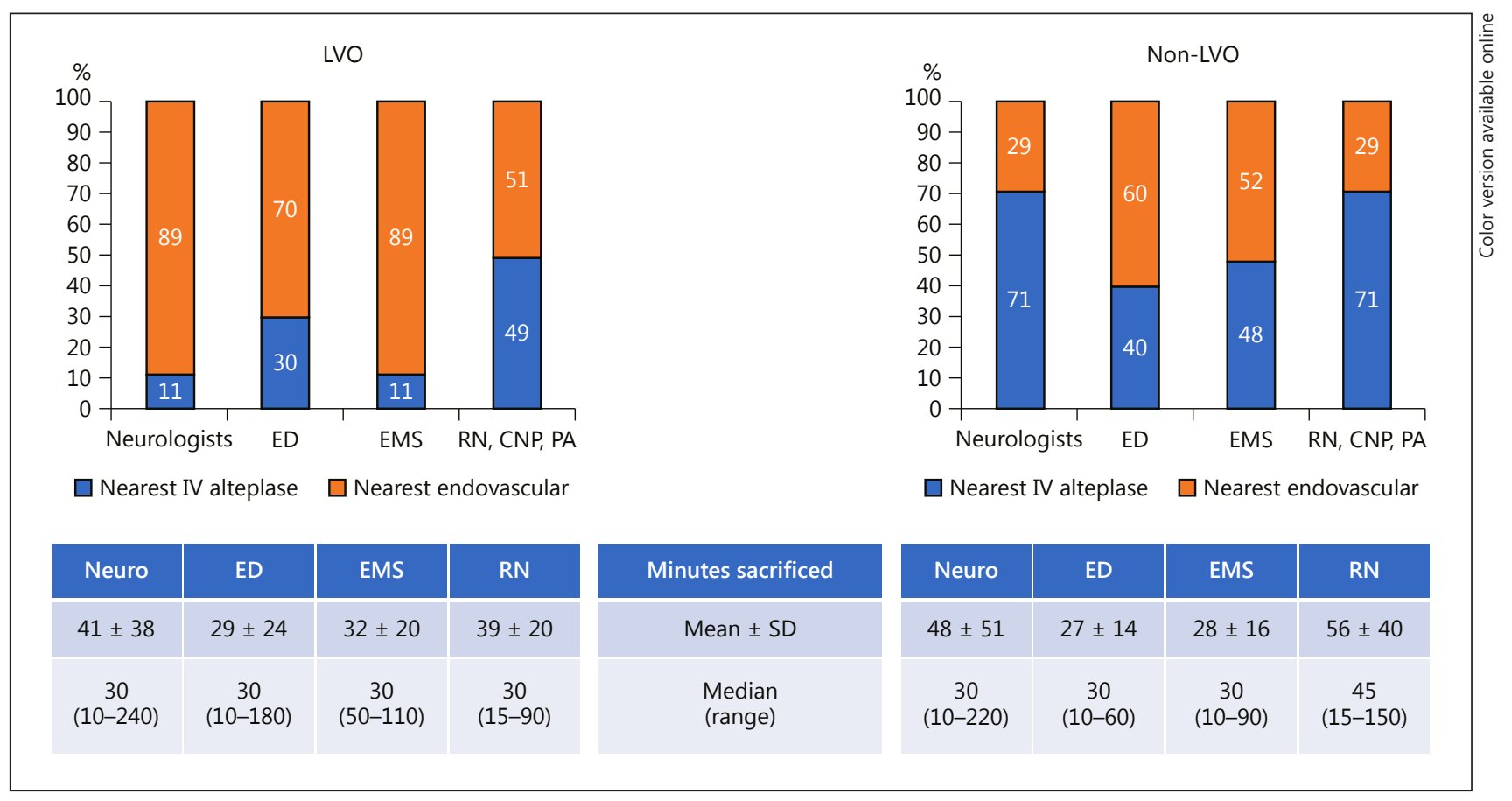

Fig. 1. Results of the triage survey by subspecialty. LVO, large vessel occlusion; Neuro, neurology; ED, emergency department; EMS, emergency medical services; RN, registered nurse (referring to stroke center coordinators including certified nurse practitioner [CNP] and physician assistant [PA]).

In response to the LVO case, $72.5 \%$ of responders chose to send the patient to ECH, whereas in the case of non-LVO stroke, $56 \%$ of responders preferred sending the patient to $\mathrm{ACH}$. When the responders were categorized according to their specialty, important differences emerged. The overwhelming majority of neurologists, $89 \%$, supported triage of the LVO case to ECH and 71\% supported triage of the non-LVO case to the ACH. Most ED and EMS physicians favored triaging both LVO and non-LVO to the ECH. Stroke coordinators had the most support for $\mathrm{ACH}$ with $49 \%$ favoring triaging $\mathrm{LVO}$ to the $\mathrm{ACH}$ and $71 \%$ favoring it in the non-LVO case. Interestingly, almost all groups agreed on the acceptable extra time to transport to ECH with a median of $30 \mathrm{~min}$ (Fig. 1).

\section{Discussion}

Despite the lack of response rate, the survey results did provide new insight as to the attitudes of current practitioners across a wide geographical area. These providers have different but close perspectives. They all deal with ischemic stroke emergencies but have different roles. Recognizing, and, sanguinely, eventually harmonizing, the diversity of viewpoints is the rationale for this research. A large majority supported bypassing ACH when LVO was suspected despite the lack of randomized controlled trial proving that such a model would improve outcomes for LVO cases and not significantly penalize non-LVO patients delayed in receiving IV tPA by mistriage. This indicates that the responders believed that endovascular therapy is the optimal treatment modality for LVO patients with IV alteplase being less or even not important at all as suggested by a recent analysis [11]. The more surprising finding was that a sizable minority, $44 \%$, supported triaging non-LVO case 
to the further ECH. Neurologists have the most internal agreement among themselves and there seems to be a clear dichotomy in triage decision based on probability of LVO. This may reflect the neurology specialty bias that LVO can be accurately differentiated from non-LVO on readily apparent clinical grounds, which is not always the case [12]. The only definitive way to exclude LVO is by vascular imaging. ED and EMS physicians preferred ECH for LVO and non-LVO cases. Whether this attitude is due to strong conviction about the benefit of endovascular therapy, fear of lack of adequate supportive care post IV thrombolysis (such as available neurocritical and neurosurgery care), or historical mistrust of alteplase is unclear. The stroke centers coordinators were the least supportive of the proposed triage model, perhaps reflecting concern about harm if IV alteplase is delayed and/or concern about ACH hospitals losing their local patients leading to deterioration of experience and practice. It is important to highlight that most patients in the endovascular arm of the positive endovascular trials (83\%) were treated with IV alteplase before endovascular treatment.

The near unanimous agreement on the accepted extra transfer time to reach the further ECH being exactly $30 \mathrm{~min}$, is another surprise. The basis of this cutoff is unknown. According to the Get-With-The-Guidelines data analysis by Saver et al. [13], a 30-min delay could lead to 8 more patients dying out of every 1,000 patients treated with IV alteplase within a $4.5-\mathrm{h}$ window. However, the effect of delay is not uniform across the $0-4.5$-h time window as can be inferred by the difference in the number needed to treat to achieve excellent outcome: 4.5 in the first $90 \mathrm{~min}$ from symptom onset to 9 in the second $90 \mathrm{~min}$ and 14 in the third $90 \mathrm{~min}$ [14].

This survey was conducted in mid-June 2017, about 5 months before the publication of the DAWN trial (DWI or CTP Assessment with Clinical Mismatch in the Triage of Wake-Up and Late Presenting Strokes Undergoing Neurointervention with Trevo) [15], and it is possible that the responders' interpretation of this pivotal trial would impact the responses. On one end, DAWN has finally ended the era of utilizing time as surrogate marker for brain viability, which may reduce the anxiety about the time window, encouraging more to choose to triage patients to the closer ACH for alteplase administration followed by transfer to ECH. On the other hand, DAWN does not negate the important effect of time on loss of salvageable brain tissue as demonstrated by ASPECTS decay [16], which would still argue with bypassing ACH in LVO patients.

Although most of the survey responders prefer triaging LVO stroke directly to the ECH, there is enough difference of opinion to declare a state of equipoise [17]. This equipoise is mainly driven by the lack of agreement in handling of non-LVO stroke, especially with the lack of a reliable tool to differentiate between the two.

\section{Conclusion}

In this survey of healthcare providers who routinely care for acute stroke patients, most responders favor bypassing hospitals without endovascular capabilities if LVO stroke is suspected, while there was disagreement on triaging patients whose strokes are not suspected to be due to LVO. This warrants a clinical trial, or a series of trials, to examine and refine different aspects of the proposed model first, then compare it with the current practice. Of critical importance is a triage tool of proven accuracy when used in the field by EMS providers. The comparative trials should be designed to show superiority rather than non-inferiority in order to drive a change in practice. 


\section{Acknowledgement}

We thank Drs Michelle Biros and Aaron Burnett for their help sending the survey to ED and EMS groups.

\section{Disclosure Statement}

Haitham M. Hussein and David C. Anderson have no disclosures.

\section{References}

1 Powers WJ, Derdeyn CP, Biller J, Coffey CS, Hoh BL, Jauch EC, et al: 2015 American Heart Association/American Stroke Association Focused Update of the 2013 Guidelines for the Early Management of Patients with Acute Ischemic Stroke Regarding Endovascular Treatment: A Guideline for Healthcare Professionals from the American Heart Association/American Stroke Association. Stroke 2015;46:3020-3035.

2 Furlan A, Higashida R, Wechsler L, Gent M, Rowley H, Kase C, et al: Intra-arterial prourokinase for acute ischemic stroke. The PROACT II study: a randomized controlled trial. Prolyse in Acute Cerebral Thromboembolism. JAMA 1999;282:2003-2011.

3 Badhiwala JH, Nassiri F, Alhazzani W, Selim MH, Farrokhyar F, Spears J, et al: Endovascular thrombectomy for acute ischemic stroke: a meta-analysis. JAMA 2015;314:1832-1843.

4 Berkhemer OA, Fransen PS, Beumer D, van den Berg LA, Lingsma HF, Yoo AJ, et al: A randomized trial of intraarterial treatment for acute ischemic stroke. N Engl J Med 2015;372:11-20.

5 Campbell BC, Mitchell PJ, Kleinig TJ, Dewey HM, Churilov L, Yassi N, et al: Endovascular therapy for ischemic stroke with perfusion-imaging selection. N Engl J Med 2015;372:1009-1018.

6 Goyal M, Demchuk AM, Menon BK, Eesa M, Rempel JL, Thornton J, et al: Randomized assessment of rapid endovascular treatment of ischemic stroke. N Engl J Med 2015;372:1019-1030.

7 Jovin TG, Chamorro A, Cobo E, de Miquel MA, Molina CA, Rovira A, et al: Thrombectomy within $8 \mathrm{~h}$ after symptom onset in ischemic stroke. N Engl J Med 2015;372:2296-2306.

8 Saver JL, Goyal M, Bonafe A, Diener HC, Levy EI, Pereira VM, et al: Stent-retriever thrombectomy after intravenous t-PA vs. t-PA alone in stroke. N Engl J Med 2015;372:2285-2295.

9 Saver JL, Goyal M, van der Lugt A, Menon BK, Majoie CB, Dippel DW, et al: Time to treatment with endovascular thrombectomy and outcomes from ischemic stroke: a meta-analysis. JAMA 2016;316:1279-1288.

10 Southerland AM, Johnston KC, Molina CA, Selim MH, Kamal N, Goyal M: Suspected large vessel occlusion: should emergency medical services transport to the nearest primary stroke center or bypass to a comprehensive stroke center with endovascular capabilities? Stroke 2016;47:1965-1967.

11 Coutinho JM, Liebeskind DS, Slater LA, Nogueira RG, Clark W, Davalos A, et al: Combined intravenous thrombolysis and thrombectomy vs thrombectomy alone for acute ischemic stroke: a pooled analysis of the SWIFT and STAR studies. JAMA Neurol 2017;74:268-274.

12 Kim JT, Park MS, Chang J, Lee JS, Choi KH, Cho KH: Proximal arterial occlusion in acute ischemic stroke with low NIHSS scores should not be considered as mild stroke. PLoS One 2013;8:e70996.

13 Saver JL, Fonarow GC, Smith EE, Reeves MJ, Grau-Sepulveda MV, Pan W, et al: Time to treatment with intravenous tissue plasminogen activator and outcome from acute ischemic stroke. JAMA 2013;309:2480-2488.

14 Lees KR, Bluhmki E, von Kummer R, Brott TG, Toni D, Grotta JC, et al: Time to treatment with intravenous alteplase and outcome in stroke: an updated pooled analysis of ECASS, ATLANTIS, NINDS, and EPITHET trials. Lancet 2010;375:1695-1703.

15 Nogueira RG, Jadhav AP, Haussen DC, Bonafe A, Budzik RF, Bhuva P, et al: Thrombectomy 6 to 24 h after stroke with a mismatch between deficit and infarct. N Engl J Med 2018;378:11-21.

16 Sun CH, Connelly K, Nogueira RG, Glenn BA, Zimmermann S, Anda K, et al: ASPECTS decay during inter-facility transfer predicts patient outcomes in endovascular reperfusion for ischemic stroke: a unique assessment of dynamic physiologic change over time. J Neurointerv Surg 2015;7:22-26.

17 London AJ: Equipoise in research: integrating ethics and science in human research. JAMA 2017;317:525-526. 